\title{
Analysis of Photoelastic Properties of Monocrystalline Silicon
}

\author{
Markus Stoehr ${ }^{1}$, Gerald Gerlach ${ }^{2}$, Thomas Härtling², Stephan Schoenfelder \\ ${ }^{1}$ Leipzig University of Applied Sciences, Faculty of Engineering, \\ Karl-Liebknecht-Str. 134, 04277 Leipzig \\ ${ }^{2} T U$ Dresden, Faculty of Electrical and Computer Engineering, Helmholtzstr. 18, 01069 Dresden
}

\begin{abstract}
Abtract
Photoelasticity could be a useful measurement tool for non-destructive, contactless determination of mechanical stresses or strains in production of silicon wafers. It describes a change in indices of refraction of materials when stressed. Since silicon has a diamond lattice structure, the stress-dependent change in refractive indices varies with loading direction. In this work an anisotropic stress-optic law is derived and compared to existing models for photoelasticity in silicon from literature.
\end{abstract}

Keywords: Photoelasticity, Monocrystalline Silicon, Infrared, Non-destructive Testing

\section{Introduction}

Photoelasticity is a contactless, non-destructive method to optically measure mechanical stresses or strains. Therefore, it offers an interesting in-line measurement method to determine and characterise transparent materials. Currently, it is employed for the production of glasses, but it could also be useful in the production process of silicon wafers. Though, while glass has an isotropic structure, silicon has an inherent mechanical and photoelastical anisotropy because of its lattice structure. Therefore, a corresponding model is required to determine the photoelastical properties of silicon.

Photoelasticity is based on birefringence caused by mechanical stress (respectively strain). Birefringence describes the property of materials to split an incident electro-magnetic wave into two refracted waves instead of one. These two refracted waves of light show different coefficients of refraction and different states of polarisation [1], [2]. A mechanical stress applied to a material susceptible to birefringence results in a change in the difference of the two states of polarisation and, hence, a change in the indices of refraction $n_{1}$ and $n_{2}$ :

$$
\Delta n=n_{1}-n_{2} .
$$

This change can be measured with a polariscope by measuring the phase difference $\delta$ between the two refracted light waves that increases with the thickness $t$ of the material:

$$
\delta=t\left(n_{1}-n_{2}\right) \text {. }
$$

For mechanically and photoelastically isotropic materials the phase difference is proportional to the difference in the first and second principal stress $\left(\sigma_{I}, \sigma_{I I}\right)$ for plane stress conditions:

$$
\delta=\operatorname{Ct}\left(\sigma_{\mathrm{I}}-\sigma_{\mathrm{II}}\right) \text {. }
$$

The stress-optic coefficient $C$ depends in general on both the material and the wavelength. It relates the difference in the principal stresses $\sigma_{I}$ and $\sigma_{I I}$ to the phase difference $\delta$.

For mechanically and photoelastically anisotropic materials, e.g. silicon, Eq. (2) does not hold true, because the lattice structure of silicon leads to a direction-dependent behaviour. In literature several different models exist for the description of the photoelastic properties of silicon. However, they lead to vastly different results (compare [3]-[5]) even when based on the same approach as shown in Fig. 1.

Therefore, in this work a new model is presented to describe the photoelastic material properties of silicon. This is based on the phenomenological approach for birefringence and photoelasticity by Pockels and Neumann [6][8].

\section{Theoretical Analysis: Photoelasticity in (100)-Silicon}

The indices of refraction due to birefringence of an unstressed material can be described by Maxwell's equations. However, this approach leads to impractically long equations. In order to simplify this, an analogy is used consisting of an ellipsoid that expresses the material properties. 


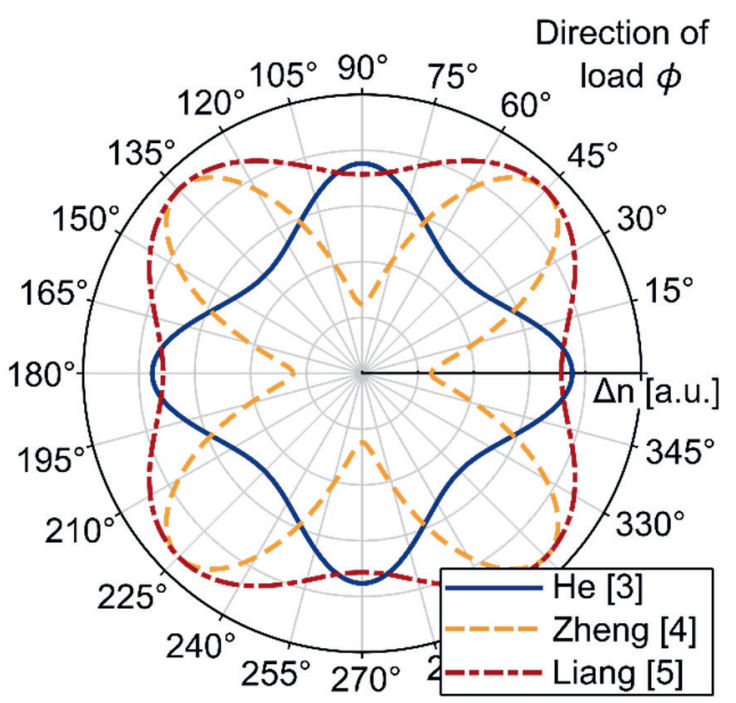

Fig. 1: Comparison of different models for the photoelasticity of silicon from literature expressed as the difference $\Delta n$ between the coefficients of refraction

In literature this ellipsoid is usually called indicatrix [9]. It is based on an imaginary light ray falling into the centre of the indicatrix. Perpendicular to this ray, a plane is constructed and the intersection of the plane with the indicatrix generates an ellipse. The lengths of the two half axis of that ellipse correspond to the two indices of refraction. This analogy is shown in Fig. 2.

The indicatrix is a quadratic surface with in general six independent parameters:

$$
\mathrm{B}_{\mathrm{ij}} x_{i} x_{j}=1 \text { for } i=(1,2,3) \text {, }
$$

where $B_{i j}$ is a $3 \times 3$ symmetric second-order tensor that depends on the material. It is called impermeability tensor. Vector $x_{i}$ represents a Cartesian coordinate system which is in this work defined so that $x_{1} \rightarrow x, x_{2} \rightarrow y$ and $x_{3} \rightarrow z$. By using the Einstein summation convention, Eq. (4) is expanded to:

$$
\begin{aligned}
& \mathrm{B}_{11} \mathrm{x}^{2}+B_{22} y^{2}+B_{33} z^{2} \\
+ & 2 B_{12} x y+2 B_{13} x z+2 B_{23} y z=1 .
\end{aligned}
$$

By rotating the indicatrix to align with the used $(x, y, z)$-coordinate system it can be expressed by only three independent values:

$$
\mathrm{B}_{\mathrm{I}} \mathrm{x}^{2}+B_{I I} y^{2}+B_{I I I} z^{2}=1 .
$$

Here, $B_{I}, B_{I I}$ and $B_{I I I}$ are the principal values of $B_{i j}$. By definition, the indicatrix can also be expressed as the reciprocal of the squared indices of refraction $\left(n_{I}, n_{I I}, n_{I I}\right)$ :

$$
\frac{1}{n_{I}^{2}} \mathrm{x}^{2}+\frac{1}{n_{I I}^{2}} y^{2}+\frac{1}{n_{I I I}^{2}} z^{2}=1,
$$

or in a more general form:

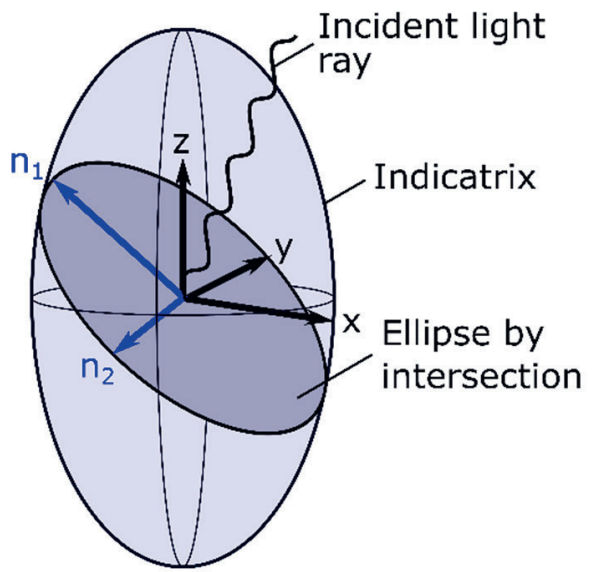

Fig. 2: Indicatrix as an analogy to describe the two indices of refraction for a certain direction of incident light in a birefringent material

$$
B_{i j}=\frac{1}{n_{i j}} .
$$

Pockels' and Neumann's phenomenological approach [6]-[8] to describe the effect of photoelasticity links the change of the impermeability $\Delta B_{i j}$ with the mechanical stress tensor $\sigma_{i j}$ and the mechanical strain tensor $\varepsilon_{i j}$, respectively, by a fourth-order tensor. In terms of the analogy of an indicatrix, this means that mechanical stresses or strains deform the indicatrix:

$$
\begin{aligned}
\Delta B_{i j} & =p_{i j k l} \sigma_{k l}, \\
\Delta B_{i j} & =q_{i j k l} \varepsilon_{k l} .
\end{aligned}
$$

Here, $p_{i j k l}$ and $q_{i j k l}$ are the stress-optical and the strain-optical tensors, respectively. Both can be expressed by each other under consideration of Hooke's law for linear elasticity [10]. Therefore, in this work only the stress-optical relationship is considered.

The change in impermeability can be expressed as the difference between the stressed and the unstressed indicatrix given by the impermeability $B_{i j}$ and $B_{i j}^{o}$ for the stressed and the undeformed indicatrices. Using Eq. (8) they can be expressed by the refraction indices $n_{i j}$ and $n_{i j}^{o}$ :

$$
\begin{gathered}
\Delta \mathrm{B}_{\mathrm{ij}}=B_{i j}-B_{i j}^{o}, \\
\Delta \mathrm{B}_{\mathrm{ij}}=\frac{1}{\left(n_{i j}\right)^{2}}-\frac{1}{\left(n_{i j}^{o}\right)^{2}} .
\end{gathered}
$$

By converting to a common denominator this can be rewritten to:

$$
\Delta \mathrm{B}_{\mathrm{ij}}=\frac{\left(n_{i j}^{o}+n_{i j}\right)\left(n_{i j}^{o}-n_{i j}\right)}{\left(n_{i j}\right)^{2}\left(n_{i j}^{o}\right)^{2}} .
$$

Since the change in impermeability can be considered small in comparison to the unstressed 
impermeability (meaning: $n_{i j} \approx n_{i j}^{o}$ ), two simplifications can be made:

$$
\begin{gathered}
\left(n_{i j}^{o}\right)^{2}+\left(n_{i j}\right)^{2} \approx 2 n_{i j}^{o}, \\
\left(n_{i j}^{o}\right)^{2}\left(n_{i j}\right)^{2} \approx\left(n_{i j}^{o}\right)^{4} .
\end{gathered}
$$

With those simplifications, Eq. (11) can be expressed as:

$$
\Delta \mathrm{B}_{\mathrm{ij}} \approx \frac{2}{\left(n_{i j}^{o}\right)^{3}}\left(n_{i j}^{o}-n_{i j}\right) .
$$

Leading to an expression in which the change between the indices of refraction of the stressed and the unstressed material is linked with the change in impermeability and, therefore, with the mechanical stress by Eq. (9a). In the following, the approximation sign is omitted, though, it still is an approximation only valid for small changes of impermeability. Rearranging Eq. (13) yields:

$$
\left(n_{i j}-n_{i j}^{o}\right)=-\frac{\left(n_{i j}^{o}\right)^{3}}{2} \Delta \mathrm{B}_{\mathrm{ij}} .
$$

For an indicatrix whose half axis are aligned to the coordinate system this leads to a set of three equations:

$$
\begin{gathered}
\left(n_{I}-n_{I}^{o}\right)=-\frac{\left(n_{I}^{o}\right)^{3}}{2} \Delta \mathrm{B}_{\mathrm{I}}, \\
\left(n_{I I}-n_{I I}^{o}\right)=-\frac{\left(n_{I I}^{o}\right)^{3}}{2} \Delta \mathrm{B}_{I \mathrm{I}}, \\
\left(n_{I I I}-n_{I I I}^{o}\right)=-\frac{\left(n_{I I I}^{o}\right)^{3}}{2} \Delta \mathrm{B}_{I I I} .
\end{gathered}
$$

With regard to Eq. (9), the change in indices of refraction can be calculated for a stress state $\sigma_{i j}$ and a known stress optic tensor $p_{i j k l}$. Because the impermeability and the stress tensors are symmetric second-order tensors, the stress-optic tensor must show certain symmetries [10]. This allows it to be written as a $6 \times 6$ matrix in Voigt notation. In this form the indices $i j$ and $k l$ of the tensor $p_{i j k l}$ are reduced to $11 \rightarrow 1$, $22 \rightarrow 2,33 \rightarrow 3,13 \rightarrow 4,23 \rightarrow 5$ and $12 \rightarrow 6$. In the following, Voigt notation will be indicated by ${ }^{(\mathrm{V})}$.

Due to the diamond structure of monocrystalline silicon there are only three independent parameters of the stress-optic tensor [10]:

$p^{(\mathrm{V})}=\left(\begin{array}{cccccc}p_{11} & p_{12} & p_{12} & 0 & 0 & 0 \\ p_{12} & p_{11} & p_{12} & 0 & 0 & 0 \\ p_{12} & p_{12} & p_{11} & 0 & 0 & 0 \\ 0 & 0 & 0 & p_{44} & 0 & 0 \\ 0 & 0 & 0 & 0 & p_{44} & p_{44} \\ 0 & 0 & 0 & 0 & 0 & 0\end{array}\right)$.

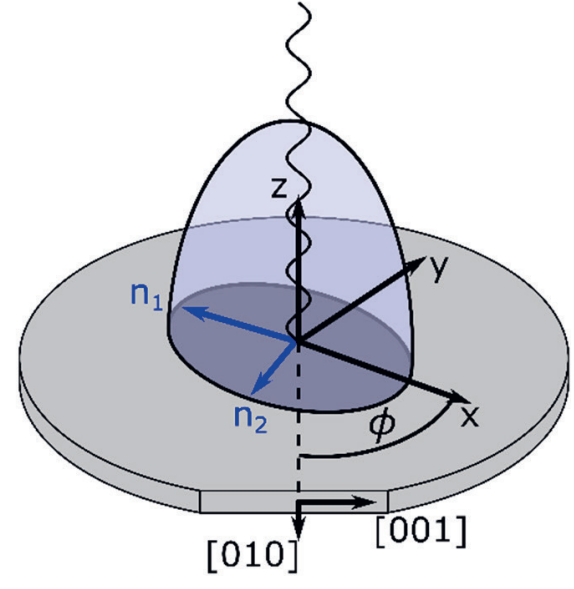

Fig. 3: $\quad$ Orientation of a (100)-Si wafer and the indicatrix describing birefringence with reference to the global $(x, y, z)$ coordinate system

In order to account for the different crystalline orientations of the silicon, a rotation matrix $R_{i j}$ is introduced. The rotated stress-optic tensor $p_{i j k l}^{\prime}$ accounts for different orientations of the silicon lattice structure by applying the rotation matrix $R_{i j}$ to it:

$$
p_{i j k l}^{\prime}=R_{\text {im }} R_{j n} R_{k o} R_{l p} p_{\text {mnop }}
$$

For simplicity, in the following only mechanical stresses in plane with the (100) plane of silicon are discussed. The $<100>$ direction is further assumed to be parallel with the z-axis of the coordinate system. A situation in which these simplifications hold true is represented by a (100)silicon wafer as shown in Fig. 3 . In that case the rotation matrix $R_{i j}(\phi)$ describing the rotation around the z-axis by an angle $\phi$ is:

$$
R_{i j}(\phi)=\left(\begin{array}{ccc}
\cos \phi & -\sin \phi & 0 \\
\sin \phi & \cos \phi & 0 \\
0 & 0 & 1
\end{array}\right) .
$$

Since the (100)-silicon wafer is considered sufficiently thin, only plane stresses are evaluated:

$$
\sigma_{i j}=\left(\begin{array}{ccc}
\sigma_{11} & \sigma_{12} & 0 \\
\sigma_{12} & \sigma_{22} & 0 \\
0 & 0 & 0
\end{array}\right)
$$

Inserting the rotation matrix of Eq. (18) into Eq. (17) and applying both to Eq. (9a) yields the impermeability tensor for the plane stress with stress tensor:

$$
\Delta B_{i j}=\left(\begin{array}{ccc}
B_{11}^{\prime}(\phi) & B_{12}^{\prime}(\phi) & 0 \\
B_{12}^{\prime}(\phi) & B_{22}^{\prime}(\phi) & 0 \\
0 & 0 & B_{33}^{\prime}(\phi)
\end{array}\right) .
$$

If the incident light ray falling into the indicatrix is parallel to the $z$-axis of the chosen coordinate system, Eq. (15c) can be neglected. Therefore, the change in the indices of refraction can be expressed by subtracting Eq. (15b) from (15a). 


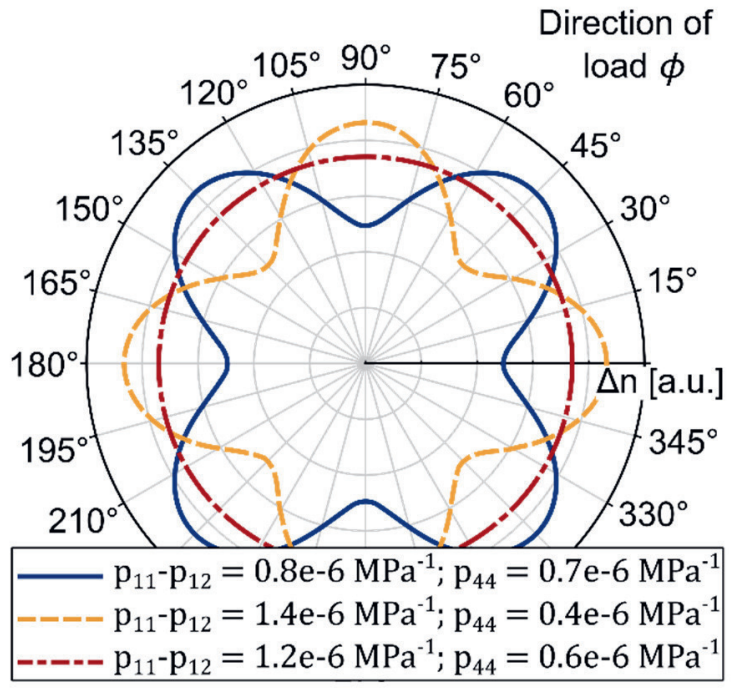

Fig. 4: $\quad$ Derived model for photoelasticity with arbitrarily chosen material parameters $p_{11}-p_{22}$ and $p_{44}$ for a plane stress state without shear stresses $\left(\sigma_{12}=0\right)$

Further, the unstressed birefringence is comparably small against the stress-induced birefringence $\left(n_{i j}^{o}=n^{o}\right)$ [11], yielding:

$$
\left(n_{I}-n_{I I}\right)=-\frac{\left(n^{o}\right)^{3}}{2}\left(\Delta \mathrm{B}_{I}-\Delta B_{I I}\right) .
$$

To obtain the principal values $\Delta B_{I}$ and $\Delta B_{I I}$ a simple eigenvalue analysis on the impermeability tensor of Eq. (20) can be performed:

$$
\begin{aligned}
\Delta \mathrm{B}_{\mathrm{I}} & =\frac{1}{4}(a-\sqrt{b+c d}), \\
\Delta \mathrm{B}_{\mathrm{II}} & =\frac{1}{4}(a+\sqrt{b+c d}),
\end{aligned}
$$

in which are:

$\mathrm{a}=2\left(\mathrm{p}_{11}+\mathrm{p}_{12}\right)\left(\sigma_{11}+\sigma_{22}\right)$,

$b=2\left(\left(p_{11}-p_{12}\right)^{2}+4 p_{44}^{2}\right)\left(4 \sigma_{12}^{2}\right.$

$$
\left.+\left(\sigma_{11}-\sigma_{22}\right)^{2}\right) \text {, }
$$

$c=2\left(\left(p_{11}-p_{12}\right)^{2}-4 p_{44}^{2}\right)$,

$d=\left(-4 \sigma_{12}^{2}+\left(\sigma_{11}-\sigma_{22}\right)^{2}\right) \cos 4 \phi$

$$
+4 \sigma_{12}\left(\sigma_{11}-\sigma_{22}\right) \sin 4 \phi \text {. }
$$

Inserting the principal values of Eq. (22) into Eq. (21) yields the stress-optic law for a (100)-silicon wafer:

$$
\Delta n=\left(n_{I}-n_{I I}\right)=\frac{\left(n^{o}\right)^{3}}{4} \sqrt{b+c d} .
$$

In this model there are only two independent variables since the difference between $p_{11}$ and $p_{12}$ cannot be separated. In Fig. 4 the derived model for photo-elasticity in silicon is shown for three arbitrarily chosen parameter combinations.

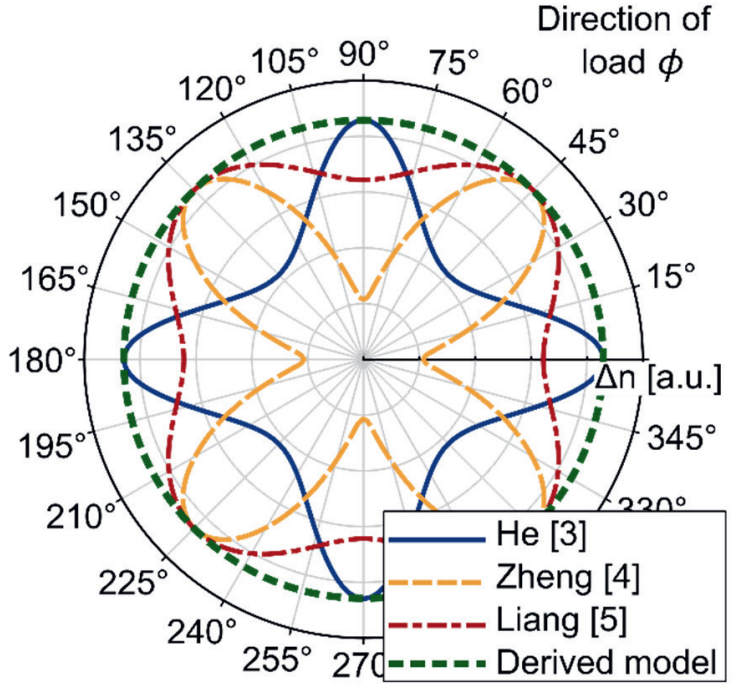

Fig. 5: $\quad$ Comparison of the derived model with models from literature for the birefringence under plane stress without shear stresses $\left(\sigma_{12}=0\right)$ for an isotropic material $\left(p_{44}=\left(p_{11}-p_{12}\right) / 2\right)$

To describe a photoelastically isotropic material the stress-optic tensor of Eq. 16 can be replaced with the stress-optic tensor for an isotropic material [10]:

$$
\begin{aligned}
& p_{i s o}^{(\mathrm{V})}= \\
& \left(\begin{array}{cccccc}
p_{11} & p_{12} & p_{12} & 0 & 0 & 0 \\
p_{12} & p_{11} & p_{12} & 0 & 0 & 0 \\
p_{12} & p_{12} & p_{11} & 0 & 0 & 0 \\
0 & 0 & 0 & \frac{p_{11}-p_{12}}{2} & 0 & 0 \\
0 & 0 & 0 & 0 & \frac{p_{11}-p_{12}}{2} & 0 \\
0 & 0 & 0 & 0 & 0 & \frac{p_{11}-p_{12}}{2}
\end{array}\right) .
\end{aligned}
$$

Following the same derivation as for the anisotropic case, the stress-optic law reduces to:

$$
\begin{aligned}
\Delta n & =\frac{\left(n^{o}\right)^{3}}{2}\left(p_{11}-p_{12}\right) \sqrt{\left(\sigma_{11}-\sigma_{22}\right)^{2}+4 \sigma_{12}^{2}} \\
& =\frac{\left(n^{o}\right)^{3}}{2}\left(p_{11}-p_{12}\right)\left(\sigma_{I}-\sigma_{I I}\right),
\end{aligned}
$$

which is the law for isotropic photo-elasticity as given in literature. The same can be achieved by replacing $p_{44}$ with $\left(p_{11}-p_{12}\right) / 2$ in Eq. (24). This special case is shown in Fig. 4 as a red dash-dotted line.

Inserting the same replacements into the models from literature shows, that those are not able to produce the isotropic case as shown in Fig. 5 whereas the newly derived model shows the expected constant difference $\Delta n$ in indices of refraction independent from loading direction $\phi$.

\section{Discussion and Conclusion}

Using Pockels' and Neumann's phenomenological approach to describe photo-elasticity in anisotropic materials, a model was derived that 
also yields the same results as derived in literature by the same approach depending on the material parameters $p_{11}-p_{12}$ and $p_{44}$. Additionally, the model is capable to describe photoelasticity in isotropic materials such as glass by choosing an appropriate combination of material parameters what is not possible with other models found in literature (compare [3]-[5]).

As a small caveat, the model is derived for a certain orientation of the crystalline diamond lattice structure in regard to the applied plane stress state and the direction of incident light. This simplification was made to allow an easy and concise determination of the principal values of the impermeability tensor $B_{i j}$. For application with different lattice orientations, the introduced rotation of the stress-optic tensor $p_{i j k l}$ can be changed accordingly and with known material parameters carried out numerically.

\section{Acknowledgement}

This work has been financially supported by the European Union as part of the ESF-Program (Project No K-7531.20/462-9), which is gratefully acknowledged. Additionally, Christiane Schuster (Fraunhofer IKTS, Germany) and Ringo Köpge (Fraunhofer IMWS, Germany) deserve thank for their support.

\section{Literature}

[1] K. Ramesh, Digital Photoelasticity - Advanced Technisques and Applications, 1st ed. SpringerVerlag Berlin Heidelberg, 2000.

[2] W. Zinth and U. Zinth, Optik, 2nd ed. München: Oldenbourg Verlag München, 2009.

[3] S. He, T. Zheng, and S. Danyluk, "Analysis and determination of the stress-optic coefficients of thin single crystal silicon samples," J. Appl. Phys., vol. 96, no. 6, pp. 3103-3109, 2004.

[4] T. Zheng and S. Danyluk, "Study of stresses in thin silicon wafers with near-infrared phase stepping photoelasticity," J. Mater. Res., vol. 17, no. 1, pp. 36-42, 2002.

[5] H. Liang, Y. Pan, S. Zhao, G. Qin, and K. K. Chin, "Two-dimensional state of stress in a silicon wafer," J. Appl. Phys., 1992.

[6] F. Pockels, Lehrbuch der Kristalloptik, 1st ed. Leipzig: B. G. Teubner, 1906.

[7] F. Pockels, "Ueber den Einfluss elastischer Deformationen, speciell einseitigen Druckes, auf das optische Verhalten krystallinischer Körper," Ann. Phys., vol. 273, no. 6, pp. 269305, 1889.

[8] K. E. Neumann, "Die Gesetze der Doppelbrechung des Lichts in comprimirten oder ungleuichförmig erwärmten unkrystallinischen Körpern," Ann. der Phys. und Chemie, vol. 54, no. 12, pp. 449-476, 1841.

[9] E. Hecht, Optik, 7. de Gruyter, 2018.

[10] T. S. Narasimhamurty, Photoelastic and Electro-Optic Properties of Crystals, 1st ed. Springer US, 1981.

[11] C. Krüger, D. Heinert and A. Khalaidovski, "Birefringence measurements on crystalline silicon," Class. Quantum Gravity, vol. 33, no. 1, 2015. 